\title{
PARSEVAL FRAMES OF PIECEWISE CONSTANT FUNCTIONS
}

\author{
DORIN ERVIN DUTKAY AND RAJITHA RANASINGHE
}

Abstract. We present a way to construct Parseval frames of piecewise constant functions for $L^{2}[0,1]$. The construction is similar to the generalized Walsh bases. It is based on iteration of operators that satisfy a Cuntz-type relation, but without the isometry property. We also show how the Parseval frame can be dilated to an orthonormal basis and the operators can be dilated to true Cuntz isometries.

Mathematics subject classification (2010): 41A30, 26A99.

Keywords and phrases: Cuntz algebras, Parseval frame, dilation.

\section{REFERENCES}

[1] Dutkay, Dorin ERvin And RAnasinghe, Rajitha, Weighted Fourier frames on self-affine measures, J. Math. Anal. Appl., 462 (2018), 1, 1032-1047, 10.1016/j.jmaa.2017.12.055, https://doi.org/10.1016/j.jmaa.2017.12.055.

[2] POPESCU, Gelu, Isometric dilations for infinite sequences of noncommuting operators, Trans. Amer. Math. Soc., 316 (1989), 2, 523-536, 10.2307/2001359, https://doi .org/10.2307/2001359.

[3] Han, Deguang, Kornelson, Keri, Larson, David and Weber, Eric, Frames for undergraduates, Student Mathematical Library, 40 American Mathematical Society, Providence, RI (2007), xiv+295, 10.1090/stml/040, https://doi.org/10.1090/stml/040.

[4] Picioroaga, Gabriel and Weber, ERic S., Fourier frames for the Cantor-4 set, J. Fourier Anal. Appl., 23 (2017), 2, 324-343, 10.1007/s00041-016-9471-0, https://doi.org/10.1007/s00041-016-9471-0.

[5] Dutkay, Dorin ERvin And Ranasinghe, Rajitha, Weighted Fourier frames on fractal measures, J. Math. Anal. Appl., 444 (2016), 2, 1603-1625, 10.1016/j.jmaa.2016.07.042, https://doi.org/10.1016/j.jmaa.2016.07.042.

[6] Dutkay, Dorin Ervin and Picioroaga, Gabriel, Generalized Walsh bases and applications, Acta Appl. Math., 133 (2014), 1-18, 10.1007/s10440-013-9856-x, https://doi.org/10.1007/s10440-013-9856-x.

[7] Dutkay, Dorin Ervin, Picioroaga, Gabriel and Song, Myung-Sin, Orthonormal bases generated by Cuntz algebras, J. Math. Anal. Appl., 409 (2014), 2, 1128-1139, 10.1016/j.jmaa.2013.07.012, https://doi.org/10.1016/j.jmaa.2013.07.012.

[8] Bratteli, O., Jorgensen, P. E. T., Kishimoto, A. And Werner, R. F., Pure states on $\mathscr{O}_{d}$, J. Operator Theory, $\mathbf{4 3}$ (2000), 1, 97-143. 